\title{
A Low Energy Routing Algorithm for the IoT Environment
}

\author{
Joonsuu Park $^{1}$, Tuvakov Jemshit ${ }^{2}$ and KeeHyun Park ${ }^{3 *}$ \\ ${ }^{1}$ Computer Engineering Dept., Keimyung University, Daegu, 42601, Rep. of Korea \\ lparkjoonsuu@gmail.com, ${ }^{2}$ jemshit.tuvakov@gmail.com, ${ }^{3 *} \mathrm{khp} @ k m u . a c . k r$
}

\begin{abstract}
In Internet of Things (IoT) environments, where resources are limited, energy is a very important resource. In an IoT environment, most nodes do not have sufficient energy and are unlikely to get chances to recharge. For this reason, one of the goals of many IoT researchers is to use energy more efficiently. In an IoT, nodes use most of their energy for communication. As such, to reduce the energy used by the IoT, it is necessary to reduce the energy used for communication. Many researchers have been making attempts in this area, but it still remains a difficult topic. In this paper, we propose algorithms that complement the energy consumed by the IoT nodes with a few variables and simple calculations. This paper also contains the results of an experimental application of the proposed algorithms.
\end{abstract}

Keywords: Internet of Things, IoT environment, Low energy routing, Energy-friendly

\section{Introduction}

Today, the fourth industrial revolution is in full swing, and is characterized by priorities such as "integration" and "connectivity". This revolution has led to the concept of the IoT (Internet of Things) that connects many objects with processors, such as sensors and actuators, rather than connecting computers. The IoT is one of the most sought-after technologies in the modern era, along with artificial intelligence, machine learning and big data, that makes the smart devices around us more "SMART" [1]. IoT is already part of our lives, as it is used in logistics, health care, smart cities and smart homes $[2,3]$.

Most devices (e.g., sensors) that are used to recognize the environment in an IoT are difficult to include in normal networks because their resources - such as power, memory, and computing power - are limited. For this reason, many researchers have studied IoT protocols that consider devices with limited resources [4-7]. Given the physical limitations of the device or the key characteristics of the IoT, IoT protocols need a way to reduce the energy used by each node for communication in order to provide a different QoS for each application and effectively increase network life. IoT protocols should be able to increase network life by efficiently using energy considering the physical limitations of the device or the main characteristics of the IoT. In most cases, each node uses most of its energy for communication. Therefore, to use energy efficiently, communication between each node must be energy-friendly, and research needs to be done in this area.

In this paper we propose a simple routing protocol that increases the efficiency of energy use in the IoT network. Based on the residual energy the node has, we used simple methods to design algorithms to ensure that as much network availability is maintained as possible.

Received (November 5, 2018), Review Result (January 5, 2018), Accepted (September 29, 2018)

${ }^{*}$ Corresponding Author 
The remainder of this paper is as follows. Section 2 introduces the relevant research, and our algorithms are suggested in Section 3. In Section 4, we measure the performance of the proposed algorithms, and Section 5 presents our conclusions.

\section{Related Works}

In this section we will describe some related research. First of all, we will talk about the THE (Table sHaring multi-path Exploration) algorithm that underpins the proposed algorithm. Next, we will describe the EEURP (Energy Efficient Unicast) routing protocol routing algorithm that works in a similar manner to the proposed method. In addition, we will describe another algorithm known as SEER that works in a similar manner to the proposed algorithm.

\subsection{Table sHaring multi-path Exploration}

The THE algorithm[8] looks for multiple paths within the entire network through three phases: connecting, sharing, and integrating.

In the connecting phase, the nodes are divided into DCN and ICN depending on whether each node is connected directly or indirectly to the server or gateway.

Figure 1 shows a simple network. IN stands for the server or gateway, the shaded 1 or 2 nodes represent DCN, and the 3 or 4 with the white background means ICN.

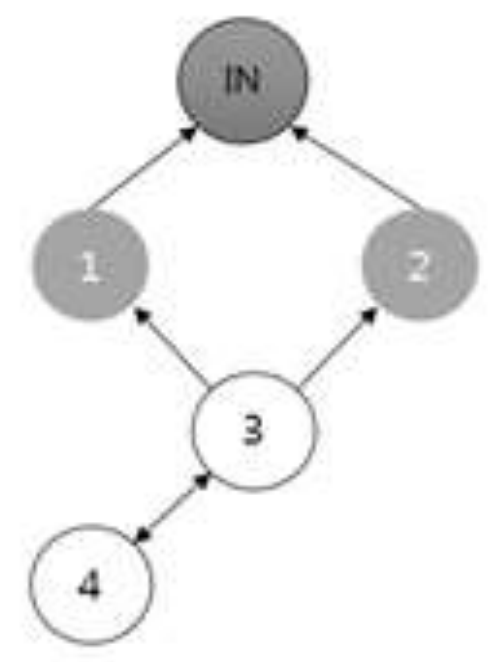

\section{Figure 1. A Simple Network}

In the sharing phase, each node generates information about all the nodes it connects to, as a regional table. Each node sends its local table to its upper node, and the upper node receiving the local table from the lower node combines the local table it receives with its own local table. This process is repeated until the server or gateway receives a local table of the entire node. The process of deduplication, table extension, link setup, and secondary deduplication of paths creates a global table for the entire network. A global table created through the above procedure has all the multiple paths of the entire network.

\subsection{Ad hoc On-demand Distance Vector (AODV) and Energy Efficient Unicast Routing Protocol (EEURP)}

EEURP is a lightweight routing protocol designed for wireless sensor networks. EEURP is built on the Ad hoc On-Demand Distance Vector (AODV) protocol proposed in RFC 3965 [9]. 
The AODV navigates the path on request. To locate the path, the AODV uses RREQ (Route Request) messages and RREP (Rout Reply) messages. If one node must find a path to another node, it will transmit (broadcast) the RREQ message to all adjacent nodes to locate the target node. When one of the adjacent nodes receives the RREQ message, it sends (broadcasts) RREQ to all of its adjacent nodes. When one of these sent RREQ messages arrives at the target node, the timer is activated and collects as many RREQ messages as possible until the timer reaches zero, then selects the path with the smallest hop count to send the RREQ for the first time. EERP is a path exploration algorithm designed to expand AODV and add minimum residual energy (MinRE) fields and total residual energy (TRE) fields within RREQ messages, taking into account minimum hop count and minimum residual energy.

\subsection{A Simple Energy Efficient Routing Algorithm for the IoT Environment}

A Simple Energy Efficient Routing Algorithm[10] is based on the THE and EEURP algorithms.

$$
\begin{gathered}
f=\frac{\sum E R}{\sum p R} \\
r=\frac{f \times p+\min E \times(1-p)}{\text { Number of Hops }}
\end{gathered}
$$

A Simple Energy Efficient Routing Algorithm calculates the $r$ value used to determine the path through the upper formula (2). The f included in formula (2) is calculated as formula (1).

$\mathrm{p}$ is the regulatory coefficient, which is also the one used in EEURP. p receives a value between 0 and 1 which determines how effective $f$ and $\operatorname{minE}$ are. In our simulation, we defined $p$ as 0.5 . Finally, minE symbolizes the minimum energy level on a route.

Like the proposed algorithm, the SEER algorithm supports transmissions that can save energy with simple calculations. However, the proposed algorithm has higher energy consideration for each node and requires simpler calculations (even calculations at the previous stage).

\section{An Energy-friendly Routing Algorithm for the IoT Environment}

The algorithm is based on our previous research, the THE algorithm. Thus, the procedure used for path exploration is the same as in the THE algorithm. Also, attributes used in the THE algorithm are still used. In addition, to take the need for energy savings into account, the energy remaining in the current node and the residual energy totals properties of that path were added to the table. 


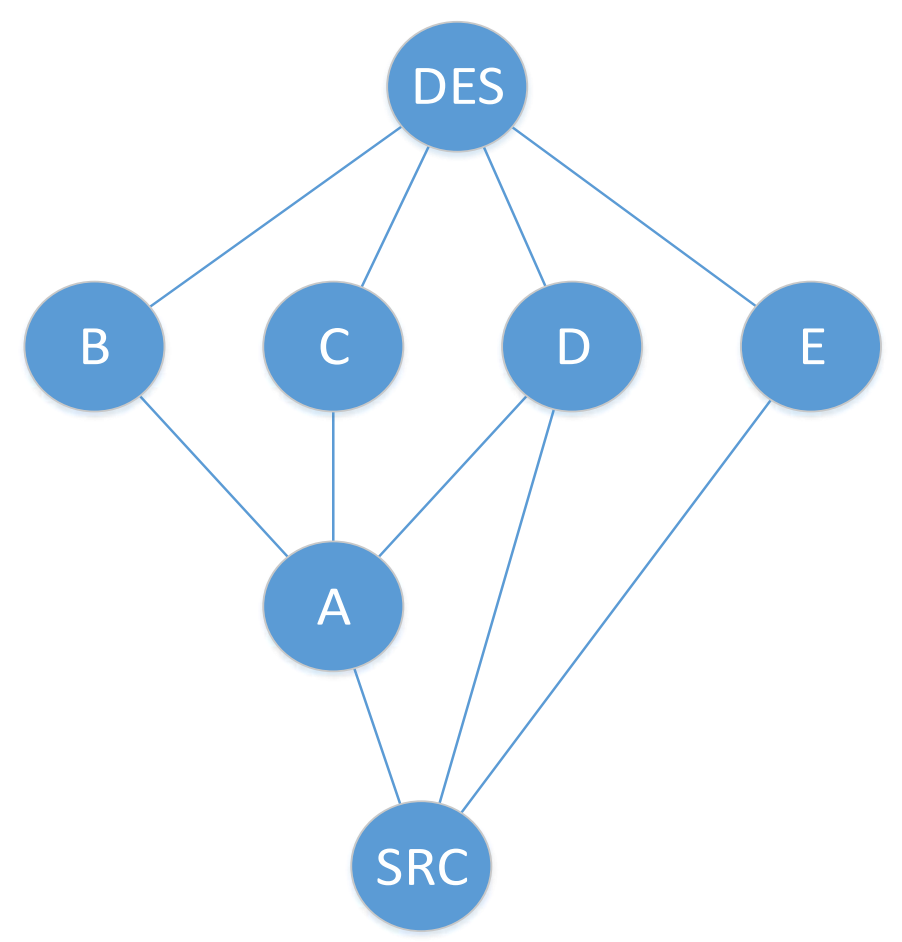

Figure 2. Network Configuration Graph \#1

Figure 2 shows a simple network diagram for use in the example. In this example, we omit part of the THE algorithm.

This algorithm performs the connection, sharing, and integration process in the same manner as the THE algorithm. Once the global table is created, the average energy value of the path is calculated and added as a field to the record. After, the priority is set according to energy consumption. A priority is used to determine the path.

In Figure 2, DES node is the final destination node and SRC node is the source node. Nodes A, B, C and D are middle nodes (path nodes).

During the integration process, SRC transfers its local tables to the nodes A, D, and E ( 1 hop-nodes). And then the receiver's local table is updated to reflect the received local table. After integration, node A sends its local table to nodes $\mathrm{B}, \mathrm{C}$ and $\mathrm{D}$, and node $\mathrm{E}$ sends to DES. To the next step, nodes B, C and D send its local table to DES.

Table 1 shows an example of the energy amount of each node.

Table 1. The Current Amount of Energy for the Nodes

\begin{tabular}{|c|c|}
\hline Nodes & Amount of energy \\
\hline A & $30 \%$ \\
\hline B & $80 \%$ \\
\hline C & $35 \%$ \\
\hline D & $40 \%$ \\
\hline E & $70 \%$ \\
\hline
\end{tabular}


If Table 1 is the current amount of energy for each node, Table 2 is the first global table created during the consolidation process.

Table 2. First Global Table

\begin{tabular}{|c|c|c|c|c|c|c|}
\hline Paths & \multicolumn{3}{|c|}{ Nodes } & Hops & $\begin{array}{c}\text { Average } \\
\text { energy }\end{array}$ \\
\hline P1 & SRC & A(30) & B(80) & DES & 1 & 55 \\
\hline P2 & SRC & A(30) & C (35) & DES & 2 & 32.5 \\
\hline P3 & SRC & A (30) & D(40) & DES & 1 & 35 \\
\hline P4 & SRC & D(40) & DES & & 2 & 40 \\
\hline P5 & SRC & E(70) & DES & & 2 & 70 \\
\hline
\end{tabular}

Next, consider the average energy and prioritize it as shown in Table 3.

Table 3. Global Table

\begin{tabular}{|c|c|c|c|c|c|c|c|}
\hline \multirow{2}{*}{ Priority } & Paths & \multicolumn{3}{|c|}{ Nodes } & Hops & Average energy \\
\hline 1 & P5 & SRC & E(70) & DES & & 1 & 55 \\
\hline 2 & P1 & SRC & A(30) & B(80) & DES & 2 & 32.5 \\
\hline 3 & P4 & SRC & D(40) & DES & & 1 & 35 \\
\hline 4 & P3 & SRC & A(30) & D(40) & DES & 2 & 40 \\
\hline 5 & P2 & SRC & A(30) & C(35) & DES & 2 & 70 \\
\hline
\end{tabular}


The following rules apply to prioritization:

- If the average energy is the same, the route with the lower hop count will have the higher priority (due to its lower overall energy consumption).

- If the hop counts are the same, the route with a lower frequency of occurrence of each node in the overall multipath will have the higher the priority.

The P5(priority 1) is selected first when sending a message. The SRC reduces the average energy value by EEV (Expected energy value). EEV is the energy value required for transmission, which can be specified as constant or the EEV field may be added during table transmit. If EEV is set as a constant 1, it means that each node consumes an average of $1 \%$ energy to transmit the message. When EEV is set to 0.5 and messages are sent 50 times, the global table changes, as shown in Table 4.

When messages are sent, average energy in the global table is updated according to EEV. If the above procedure is repeated multiple times, the priority may change, as shown in Table 4 (the number in the brackets is the energy remaining).

Table 4. Global Table Updated 50 Times

\begin{tabular}{|c|c|c|c|c|c|c|c|}
\hline Priority & Paths & \multicolumn{3}{|c|}{ Nodes } & Hops & Average energy \\
\hline 1 & P5 & SRC & E(53.5) & DES & & 53.5 & 1 \\
\hline 2 & P1 & SRC & A(24.5) & B(74.5) & DES & 49.5 & 2 \\
\hline 3 & P4 & SRC & D(29) & DES & & 29 & 1 \\
\hline 4 & P2 & SRC & A(24.5) & C(29.5) & DES & 27 & 2 \\
\hline 5 & P3 & SRC & A(24.5) & D(29) & DES & 26.75 & 2 \\
\hline
\end{tabular}

\section{Performance Evaluation}

We conducted several tests to assess the performance of the proposed routing algorithm. We expected the proposed algorithm to consume energy more efficiently, extending the entire network life. To ensure that the expected goals are met, sequential transmission and algorithm performance are compared within the node composition of Figure 2, Figure 3, and Figure 4. In addition, transmission is stopped if an unavailable path occurs among multiple paths. This is a way to measure network life. As well, to measure the network life, the transmission was set to stop if an unavailable path occurred among several paths.

Figure 3 shows a model that is expected to have equal or similar performance to sequential transmission. 


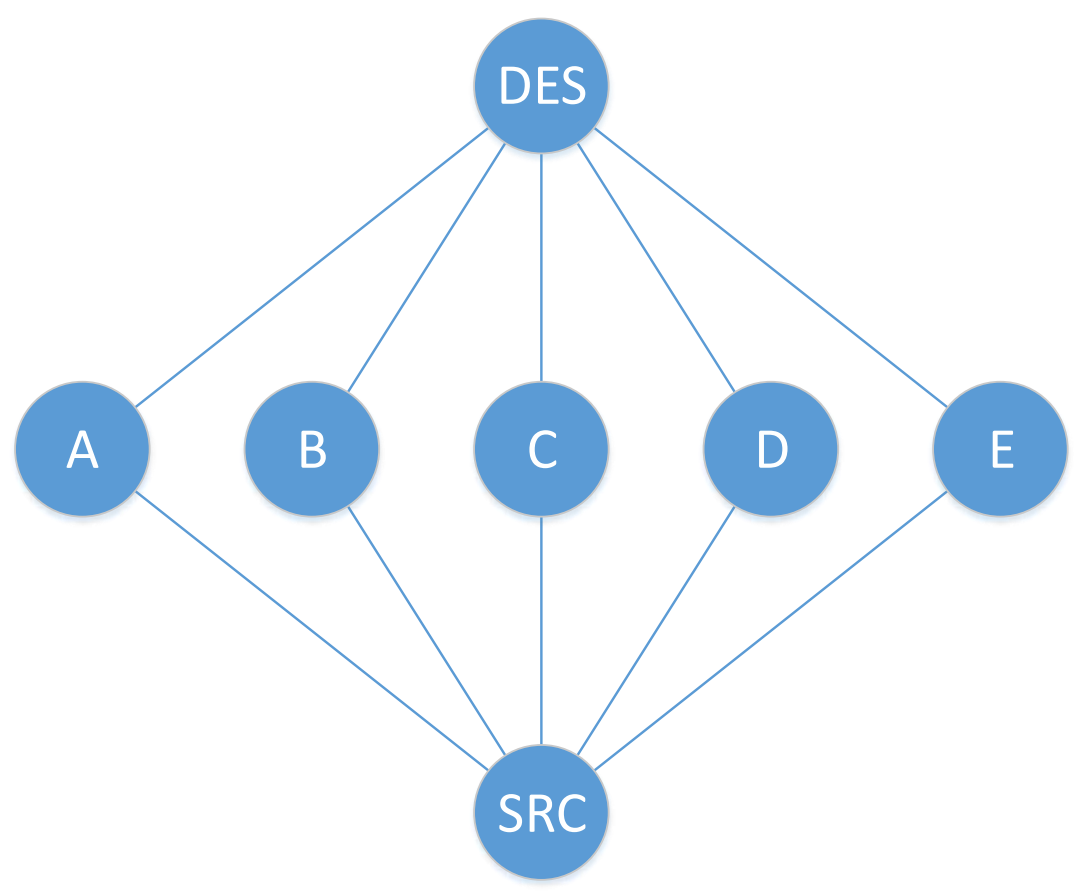

Figure 3. Network Configuration Graph \#2

Figure 3 shows the model of a complex path that is expected to have the best performance over sequential transfers.

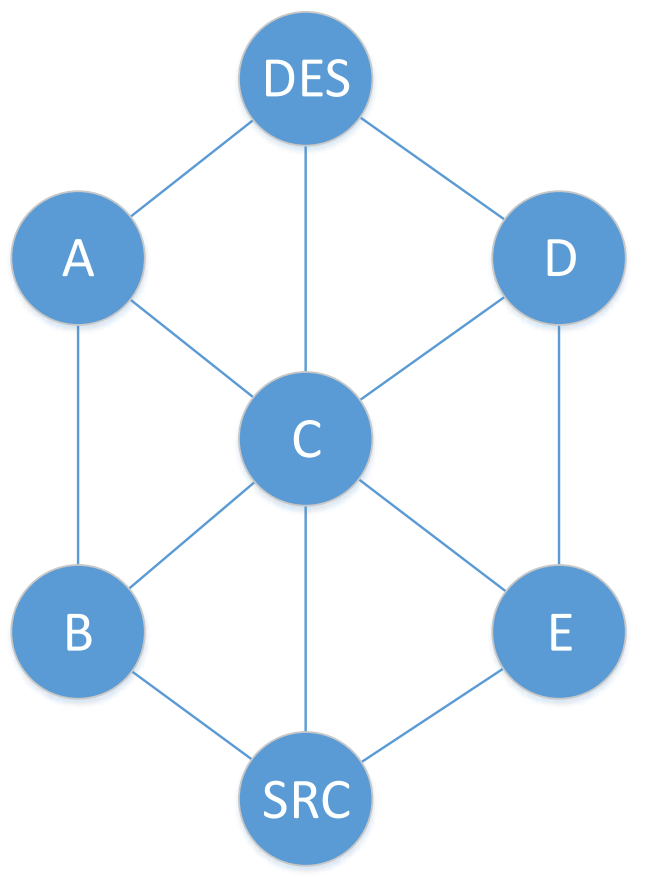

Figure 4. Network Configuration Graph \#3

We set EEV to 0.1 for the above three models and then conducted an experiment.

Figure 5 below shows the performance measurement results for the three models above. 


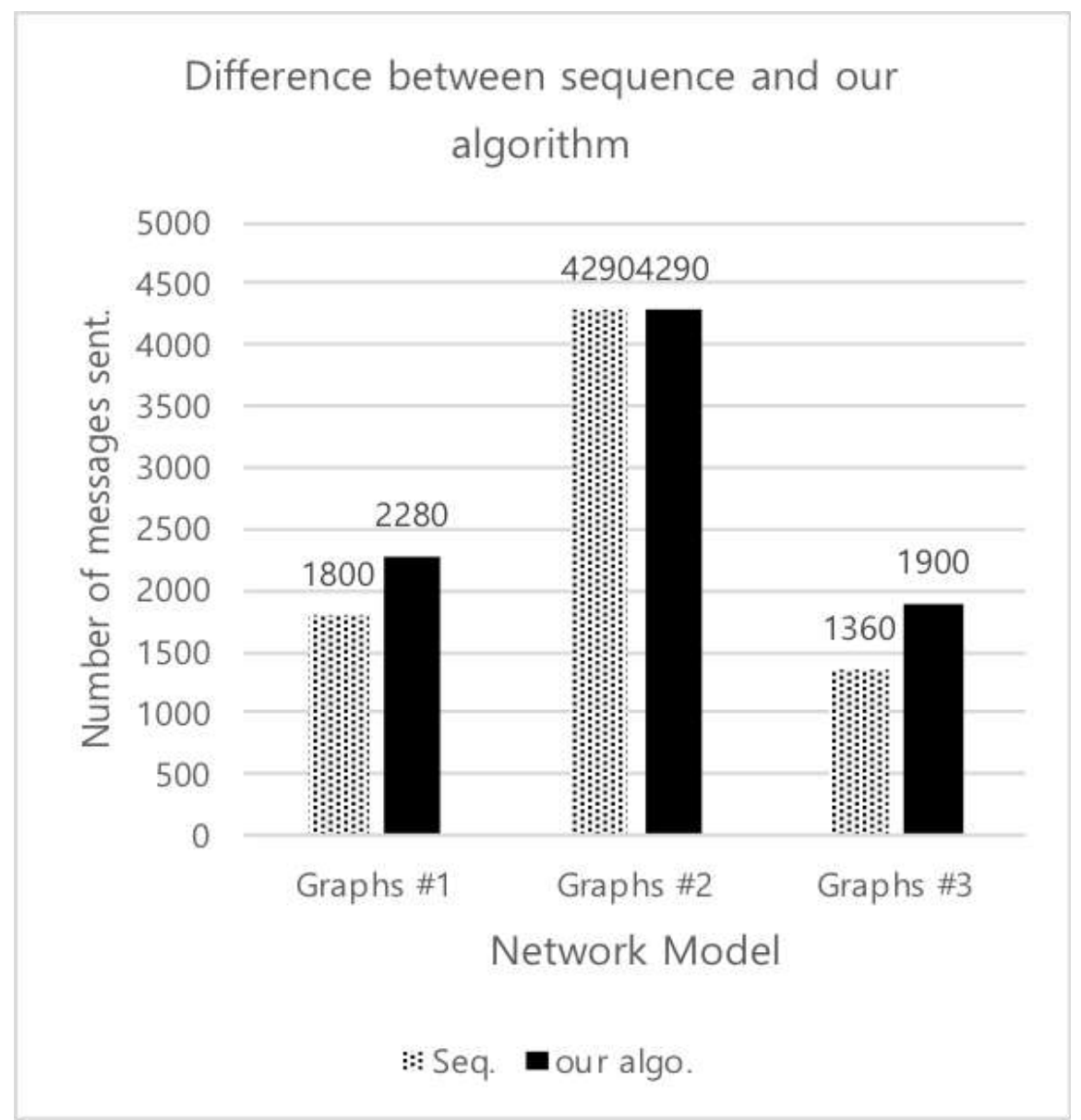

\section{Figure 5. Differences in Performance between Sequential Transmission and our Algorithm}

In the experiment, because the prioritization results in the ERR (Energy-friendly Route) algorithm are the same as the sequential routing results, graph \#2 does not differ from the sequential transmission and ERR algorithm, as expected.

Graph \#3 models have many duplicate nodes (In particular, node C). There are no nodes that belong to only one path. In this model, the ER algorithm had approximately a $40 \%$ longer network life compared to a sequential algorithm. Graph \#1 is a moderate model for two models (Graph \#2, Graph \#3). In this model, the ER algorithm had approximately $27 \%$ longer network life compared to a sequential algorithm.

As a result, the more redundant nodes in a multipath, the more efficient the ER algorithm.

\section{Conclusion}

In this study, we proposed the ER Algorithm, which allows a more effective selection of routes among the detected multiple paths by expanding the THE algorithm to explore multiple paths that exist within the IoT network. Unlike the THE algorithm, which searches only paths, ER is designed to use the least energy-consuming path based on the THE's functions, with simple calculations.

The proposed algorithm can extend the life of the entire network through a simple calculation of the remaining energy in each node, the amount of energy consumed in the transmission, and the average remaining energy calculations. We did some experiments to prove this. If more nodes are involved in multiple paths, the network life is reduced, as well as the number of available paths. 


\section{Acknowledgments}

This research was supported by the Basic Science Research Programs through the National Research Foundation of Korea (NRF), funded by the Ministry of Education, Science and Technology (No. NRF-2018R1D1A1B07043982).

\section{References}

[1] P. Sethi and S. R. Sarangi, "Internet of things: architectures, protocols, and applications", Journal of Electrical and Computer Engineering, vol. 2017, (2017).

[2] X. Mao, K. Li, Z. Zhang and J. Liang, "Design and implementation of a new smart home control system based on internet of things", in Smart Cities Conference (ISC2), 2017 International, IEEE, (2017), pp. 15 .

[3] A. M. Rahmani, "Exploiting smart e-Health gateways at the edge of healthcare Internet-of-Things: A fog computing approach," Future Generation Computer Systems, vol. 78, (2018), pp. 641-658.

[4] A. M. Ortiz, F. Royo, T. Olivares, N. Timmons, J. Morrison and L. Orozco-Barbosa, "Intelligent routing strategies in wireless sensor networks for smart cities applications", Networking, Sensing and Control (ICNSC), 2013 10th IEEE International Conference on IEEE, (2013), pp. 740-745.

[5] J. Vasseur and D. Culler, "Routing over low power and lossy networks", ed, (2009).

[6] J.-Y. Chang and P.-H. Ju, "An energy-saving routing architecture with a uniform clustering algorithm for wireless body sensor networks", Future Generation Computer Systems, vol. 35, (2014), pp. 128-140.

[7] K. Machado, D. Rosário, E. Cerqueira, A. A. Loureiro, A. Neto and J. N. de Souza, "A routing protocol based on energy and link quality for internet of things applications", sensors, vol. 13, no. 2, (2013), pp. 1942-1964.

[8] J. Park, "A study on scheduling algorithm and multi-path exploration algorithm for PHD Networks", M. Sc., Keimyung, Daegu, (2016).

[9] K. Toyoda, H. Ohno, J. Murai and D. Wing, "A simple mode of facsimile using internet mail", 20701721, (2004).

[10] T. Jemshit, "A Simple Energy Efficient Routing Algorithm for the IoT Environment", (2017), pp. 7-12. 
International Journal of Control and Automation

Vol. 11, No. 10 (2018) 